\title{
Prediction of gestational weight gain - a biopsychosocial model
}

\author{
Emily Heery ${ }^{1, *}$, Cecily C Kelleher ${ }^{1}$, Patrick G Wall ${ }^{1}$ and Fionnuala M McAuliffe ${ }^{2}$ \\ ${ }^{1}$ School of Public Health, Physiotherapy and Population Science, Woodview House, University College Dublin, \\ Belfield, Dublin 4, Republic of Ireland: ' UCD Obstetrics and Gynaecology, School of Medicine and Medical Science, \\ University College Dublin, National Maternity Hospital, Dublin, Republic of Ireland
}

Submitted 26 July 2013: Final revision received 27 April 2014: Accepted 13 July 2014: First published online 29 August 2014

\begin{abstract}
Objective: To examine the influence of health behaviours and psychological well-being on gestational weight gain using a biopsychosocial model.

Design: A prospective cohort study of pregnant women consecutively recruited at their first antenatal care visit. A self-administered questionnaire was used to collect data on health behaviours and psychological well-being in early pregnancy. Linear regression and logistic regression were used to identify predictors of total weight gain in kilograms and weight gain outside the current Institute of Medicine recommendations, respectively.

Setting: A maternity hospital in the Republic of Ireland.

Subjects: Data on 799 women were analysed.

Results: Pre-pregnant BMI $\geq 30 \cdot 0 \mathrm{~kg} / \mathrm{m}^{2}$, short stature, parity $>0$, decreased food intake and absence of health insurance predicted lower absolute gestational weight gain, while foreign nationality, consumption of takeaway meals more than once weekly and increased food intake predicted higher absolute gestational weight gain. Overweight and obesity, foreign nationality, increased food intake and height $>170 \mathrm{~cm}$ were risk factors for excessive weight gain, while antenatal depression was protective against excessive weight gain. Notably, physical activity measures were not related to the gestational weight gain outcomes. Pre-pregnancy overweight and increased food intake were the strongest predictors of excessive gestational weight gain.

Conclusions: None of the psychological well-being measures examined, with the exception of antenatal depression, was associated with any of the weight gain outcomes. The behavioural predictors of gestational weight gain were increased food intake and takeaway consumption. Public health promotions should target pre-pregnancy BMI and pregnancy-associated change in food intake.
\end{abstract}

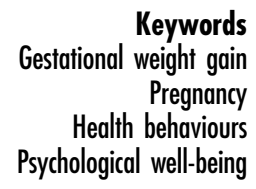

The Institute of Medicine (IOM) provides recommendations for weight gain during pregnancy according to prepregnancy BMI: $12.5-18.0 \mathrm{~kg}$ for underweight women; $11.5-16.0 \mathrm{~kg}$ for normal-weight women; $7 \cdot 0-11.5 \mathrm{~kg}$ for overweight women; and $5 \cdot 0-9 \cdot 0 \mathrm{~kg}$ for obese women ${ }^{(1)}$. Weight gains within these guidelines are associated with better maternal and child outcomes. For example, there is consistent evidence that excessive prenatal weight gain confers a greater risk for macrosomia, caesarean delivery and postpartum weight retention, while inadequate prenatal weight gain confers a greater risk for preterm birth and low birth weight ${ }^{(2,3)}$. There is also mounting evidence linking gestational weight gain (GWG) with childhood obesity ${ }^{(4)}$. The adverse health outcomes associated with GWG outside recommendations highlight the importance of examining its determinants. In turn, such research can inform public health strategies to tackle excessive and inadequate GWG.
Numerous studies have found that pre-pregnancy underweight and obesity and multiparity are independent risk factors for low $\mathrm{GWG}^{(5,6)}$, while high prepregnancy BMI and nulliparity are independent risk factors for excessive $\mathrm{GWG}^{(5-8)}$. Although the bulk of the literature focuses on biological and sociodemographic influences on GWG, increasingly researchers are examining behavioural and affective influences, as these are more amenable to intervention during the course of pregnancy. There is also a growing consensus that GWG is multifactorial in nature ${ }^{(1)}$. Indeed, some investigators have argued that GWG may be more usefully conceptualised using a biopsychosocial model, which explicitly recognises the individual and interacting influences of biomedical, psychological and social factors ${ }^{(9-11)}$.

Several reviews have highlighted numerous gaps and inconsistencies in the evidence base regarding the impact 
of behavioural and psychological well-being factors on $\mathrm{GWG}^{(1,9,12,13)}$. In particular, the IOM concluded that depressive symptoms are associated with both low and high GWG, but that the evidence on whether and how other measures of psychological well-being impact on GWG is inconclusive ${ }^{(1)}$. In addition, many reviews have highlighted that there is a lack of evidence relating dietary intake or physical activity to GWG, even though these are primary determinants of body weight in non-pregnant women ${ }^{(1,12,13)}$. Furthermore, there is a lack of evidence linking sleep to $\mathrm{GWG}^{(14)}$ and while some studies have shown a link between prenatal smoking and inadequate weight gain ${ }^{(6,15)}$, others have not ${ }^{(16,17)}$. In order to address these limitations, we aimed to examine the impact of health behaviours and psychological well-being on GWG using a biopsychosocial model. We addressed the following research question: Are potentially modifiable health behaviour and psychological well-being factors associated with GWG, when relatively unmodifiable, biological and sociodemographic factors are also taken into account? By identifying modifiable factors associated with weight gain in a biopsychosocial model, the present study may usefully inform prenatal advice and interventions.

\section{Methods}

\section{Study design}

A prospective cohort study, with institutional ethical approval, was conducted with women attending the National Maternity Hospital, Dublin, Ireland. A team of research assistants consecutively recruited patients waiting to attend their antenatal 'booking' appointment over a 3-month period (March-May 2011). Each woman was informed about the advantages of participating in the study and what participation involved for her. The eligibility of each patient to participate in the research was also ascertained. The women were eligible for recruitment if they were: (i) 18 years of age or above; (ii) able to give full informed consent; and (iii) expecting a singleton pregnancy. After providing written informed consent, the participants were given a self-completion questionnaire to obtain information in relation to psychological well-being, health behaviours and sociodemographic characteristics. The women completed the questionnaire at a mean of 15.4 (SD 3.9) weeks' gestation. Following pregnancy, biomedical information (including serial weight measurements) was obtained from the women's medical records.

\section{Measures of gestational weight gain}

Three GWG outcome measures were calculated. First, a continuous measure of total GWG was calculated by subtracting self-reported pre-pregnancy weight from the last measured weight before delivery. Next, we compared the women's total weight gain against their expected weight gain based on the IOM (2009) weight gain recommendations to create two categorical variables: (i) excessive weight gain $v$. adequate or inadequate; and (ii) inadequate weight gain $v$. adequate or excessive ${ }^{(1)}$. The calculation of the categorical outcomes adjusted for the timing of the last weight measurement before delivery and length of gestation to prevent confounding by these factors. A similar approach has been used in previous studies $^{(11,18)}$. The trimester-specific cut-offs recommended by the $\mathrm{IOM}^{(1)}$ were used to adjust the categorical outcomes.* If the woman's total weight gain fell within the upper and lower cut-offs of expected weight gain at the time of the last weight measurement in pregnancy, it was classified as adequate. Similarly, if the woman's total weight gain was above the upper cut-off of expected weight gain, it was classified as excessive and if it was below, it was deemed inadequate. Women who did not have a weight recorded within 10 weeks of delivery were excluded from the analysis. In the final analysis sample, $95 \%$ of women had a weight recorded within 4 weeks of delivery.

\section{Assessment of exposures}

\section{Biological variables}

Maternal age (18-24 years, 25-29 years, 30-34 years, 35 + years), parity $(0,1,2+)$ and height $(<157 \mathrm{~cm}, 157-$ $170 \mathrm{~cm},>170 \mathrm{~cm}$ ) were extracted from the women's medical records. Pre-pregnancy BMI $\left(\mathrm{kg} / \mathrm{m}^{2}\right)$ was

* For each woman, the lower and upper cut-offs of expected weight gain at the gestational age of the last weight measurement before delivery were calculated using the following formulae:

Lower cut-off $=$ lower cut-off of recommended first-trimester weight gain $+[$ (gestational age at last measured weight -13 weeks $)$ $\times$ lower cut-off rate of weight gain recommended for the second and third trimesters].

Upper cut-off $=$ upper cut-off of recommended first-trimester weight gain $+[$ (gestational age at last measured weight -13 weeks $)$ $\times$ upper cut-off rate of weight gain recommended for the second and third trimesters].

The lower cut-off rate of recommended weight gain for the second and third trimesters was calculated for each BMI category by subtracting the lower cut-off of first-trimester recommended weight gain from the lower cut-off of total recommended weight gain and then dividing the answer by 27 (the number of weeks in the second and third trimesters). Similarly, the upper cut-off rate of recommended weight gain for the second and third trimesters was calculated for each BMI category by subtracting the upper cut-off of first-trimester recommended weight gain from the upper cut-off of total recommended weight gain and then dividing the answer by 27 . The lower cut-offs recommended for first-trimester weight gain were, 1, 1, 1 and $0.5 \mathrm{~kg}$, and the upper cut-offs were $3,3,3$ and $2 \mathrm{~kg}$, for underweight, normal-weight, overweight and obese women, respectively ${ }^{(1,19)}$. Furthermore, the lower cut-offs recommended for total weight gain were $12.5,11.5,7$ and $5 \mathrm{~kg}$, and the upper cut-offs were $18,16,11.5$ and $9 \mathrm{~kg}$, for underweight, normal-weight, overweight and obese women, respectively $^{(1,19)}$. For example, the lower and upper cut-offs of expected total weight gain for an obese woman, whose last weight measurement was taken at 36 weeks' gestation, were calculated as follows:

$$
\begin{aligned}
& \text { Lower cut-off }=0 \times 5+[(36-13) \times(5-0 \times 5) / 27)=4 \times 33 \mathrm{~kg} \\
& \text { Upper cut-off }=2+[(36-13) \times(9-2) / 27]=7 \times 96 \mathrm{~kg} .
\end{aligned}
$$


calculated based on measured height and self-reported pre-pregnancy weight. The women were classified based on the $\mathrm{WHO}^{(20)}$ and the $\mathrm{IOM}^{(1)}$ categories as underweight $\left(<18.5 \mathrm{~kg} / \mathrm{m}^{2}\right)$, normal weight $\left(18.5-24.9 \mathrm{~kg} / \mathrm{m}^{2}\right)$, overweight $\left(25 \cdot 0-29.9 \mathrm{~kg} / \mathrm{m}^{2}\right)$ or obese $\left(\geq 30 \cdot 0 \mathrm{~kg} / \mathrm{m}^{2}\right)$. At the first hospital antenatal visit, maternal height was measured to the nearest $0 \cdot 1 \mathrm{~cm}$ using a wall-mounted stadiometer. In the questionnaire, participants were asked, 'How much did you weigh (without clothes) just before you got pregnant?' A tight correlation between measured pre-pregnancy weight and self-reported pre-pregnancy weight has been observed, with coefficients ranging from 0.96 to $0.99^{(21-23)}$.

For $8 \%$ of the analysis sample, an imputed weight was used instead of the self-reported weight, because it was missing or considered biologically implausible. Pre-pregnancy weight was imputed using the first weight measurement taken during antenatal care (if taken before 19 weeks' gestation) minus the recommended amount of weight to be gained in the first and second trimesters, as defined by the IOM.* If the first weight measurement was taken after 18 weeks' gestation, a pre-pregnancy weight was not imputed. A similar methodology for imputation of prepregnancy weight has been used previously ${ }^{(24,25)}$. Following imputation, the validity of the pre-pregnancy weight variable was checked in sample of 282 women with a weight measurement in the first trimester. The difference between the measured weight in the first trimester and the self-reported weight varied by gestational age at the time of the first weight measurement (as expected), but did not vary by BMI, nationality, parity or health insurance status.

\section{Sociodemographic variables}

Information on marital status (married, single), nationality (Irish, foreign), educational attainment (less than second level, second level, vocational/training course, degree/ postgraduate), employment status (not employed, parttime employed, full-time employed), living with the baby's father (yes, no) and private health insurance status (yes, no) was obtained from the prenatal questionnaire and the women's medical records. In addition, pregnancy intention was assessed in the questionnaire using an item taken from the Pregnancy Risk Assessment Monitoring System (PRAMS) survey ${ }^{(26)}$. The women were asked: 'Thinking back to just before you got pregnant, how did you feel about becoming pregnant?' Women were divided into two categories based on their responses. A woman's pregnancy was categorised as intended if she responded, 'I wanted to be pregnant sooner' or 'I wanted to be pregnant then'. If the woman responded, 'I wanted to be pregnant later' or 'I didn't want to be pregnant then or at any time in the future', her pregnancy was classified as unintended.

* See Rasmussen and colleagues ${ }^{(19)}$ for information on the IOM firsttrimester weight gain recommendations by BMI category that were used for imputation purposes.

\section{Psychological well-being variables}

Four measures of psychological well-being were assessed in the prenatal questionnaire using previously validated instruments. Depressive symptoms were measured using the tenitem version of the Edinburgh Postpartum Depression Scale (EPDS), which has been validated for use with pregnant and postpartum women ${ }^{(27-29)}$. Perceived level of stress during pregnancy was measured using the ten-item version of the Perceived Stress Scale, which has adequate reliability and convergent validity ${ }^{(30,31)}$.

The Prenatal Distress Questionnaire was used to measure worries and concerns specific to pregnancy, including concerns about pregnancy complications, labour and delivery, the health of the baby, bodily changes, physical symptoms, changing relationships and parenting. This twelve-item scale has been to found to have high internal consistency $^{(32)}$, adequate convergent validity with general stress measures ${ }^{(32)}$ and good predictive validity ${ }^{(33)}$. The Life Orientation Test-Revised was used to measure optimism ${ }^{(34)}$. This scale consists of ten items, three of which are fillers. It has high reliability, adequate discriminant validity and good predictive validity ${ }^{(33,34)}$.

EPDS scores above 12 were used to identify probable antenatal depression in the present research ${ }^{(35)}$. Total scores on the other three well-being scales were categorised into tertiles, as these scales have no published clinical cut-offs.

\section{Health behaviours}

Using information from the prenatal questionnaire, four health behaviours were examined in the present research: food intake, physical activity, sleep and smoking.

Several aspects of food intake behaviour were examined. Pregnancy-associated change in food intake was assessed using a modified version of an item originally used by Olson and Strawderman ${ }^{(6)}$. In the present research, the women were asked, 'How has the amount of food you eat now changed compared with times when you were not pregnant?' The five response categories were 'a lot less food now', 'a little less food now', 'about the same', 'a little more food now' and 'a lot more food now'. The original item used by Olson and Strawderman ${ }^{(6)}$ had four response categories, but was modified to include a fifth category: 'about the same'. In addition, daily energy intake in kilocalories and alcohol use during pregnancy were assessed using a 149-item $\mathrm{FFQ}^{(36)}$ that was adapted from an instrument used in the European Prospective Investigation into Cancer and Nutrition ${ }^{(37)}$. The adapted FFQ has been validated for an Irish adult population using food diaries and a protein biomarker ${ }^{(38)}$. Daily energy intake was assessed in continuous form, as well as in tertile categories. Alcohol intake in pregnancy was assessed using a dichotomous variable (yes, no). Other aspects of food intake were assessed using additional behavioural questions, including frequency of takeaway and fried food consumption and number of snacks eaten per day. 
Leisure-time exercise was assessed using the Godin Leisure-Time Exercise Questionnaire ${ }^{(39)}$, which has high validity and reliability ${ }^{(39-42)}$. A total exercise index was calculated by multiplying weekly frequencies of mild, moderate and strenuous exercise by 9, 5 and 3 MET, respectively (where MET = metabolic equivalents of task) and then summing these components to derive a total score. This score was then converted into tertile categories for analysis purposes.

Additionally, change in physical activity levels since becoming pregnant was assessed using the following item: 'How does the amount of physical activity you are getting now compare with your physical activity level before you got pregnant?' The five response categories were 'much less active now', 'a little less active now', 'about the same', 'a little more active now' and 'much more active now'. A similar measure of pregnancy-associated change in physical activity has been previously associated with frequency of pre-pregnancy exercise, pre-pregnancy BMI, exercise self-efficacy and $\mathrm{GWG}^{(6,43)}$

Sleep duration during pregnancy was measured using the following item: 'In the past month, how many hours of sleep do you get in an average 24-hour period?' The responses were dichotomised into $<7$ and $\geq 7 \mathrm{~h} / \mathrm{d}$ on the basis of prior research which found that fewer than $7 \mathrm{~h}$ of sleep is positively associated with obesity ${ }^{(44)}$.

Finally, smoking status was coded as current, if the participant reported continuing to smoke during pregnancy. In addition, smoking status was coded as former, if the participant reported quitting smoking since beginning pregnancy or in the 6 months prior to pregnancy and it was coded as non-smoker, if the participant reported quitting smoking more than 6 months before her pregnancy.

\section{Statistical analysis}

First, a series of linear regression models was used to individually examine the relationship between each of the potential risk factors and total GWG in kilograms. Next, a series of binary logistic regression models was used to individually examine the relationship between each of the potential risk factors and the odds of gaining above the current IOM guidelines $v$. not gaining above the guidelines ${ }^{(1)}$. Similarly, binary logistic models were used to individually assess the relationship between each of the predictor variables and the odds of gaining below the IOM guidelines $v$. not gaining below the guidelines. Only variables with an associated $P$ value $<0.15$ were considered for inclusion in the final regression models.

Backward stepwise methods were used to explore the multivariable associations between the predictor variables retained from the univariable analysis and the outcome measures of GWG. Simultaneous entry regression was used for the final multivariable models, as the biopsychosocial model calls for biological, psychological and social variables to be examined simultaneously. Final model selection was guided by the authors' knowledge of the relevant literature, the conceptual framework and the exploratory findings of the stepwise regression models $^{(45,46)}$. Only statistically significant variables $(P<0.05)$ were included in the final models.

Two control variables were included in each linear regression model predicting total GWG: (i) total weeks of gestation; and (ii) the number of weeks between the last measured maternal weight and delivery. These control variables were not included in the logistic regression models, as the calculation of the weight gain adequacy outcomes controlled for the length of gestation and the timing of the last weight measurement before delivery.

All analyses were conducted using the statistical software package IBM SPSS Statistics version 20.

\section{Results}

Of 1229 women invited to participate, 984 women agreed to participate and completed the study questionnaire, resulting in an $80 \%$ response rate. We excluded seventyeight women, as they had gestational diabetes or diabetes mellitus, twins, miscarriage, or changed hospital. We then excluded 107 women with insufficient maternal weight information. Of these, ninety-two women had $>10$ weeks between the last recorded weight in pregnancy and delivery, twelve women's medical chart was unavailable and two women had a missing self-reported pre-pregnancy weight and a pre-pregnancy weight was not imputable. Following all exclusions, a final sample of 799 women was available for analysis. The biological, sociodemographic, behavioural and well-being characteristics of the 799 women included in the analysis and the 107 women excluded due to insufficient weight information were compared using logistic regression. The results showed that the women excluded from the analysis were more likely to not have health insurance $(\mathrm{OR}=1.75 ; 95 \%$ CI $1.15,2 \cdot 66)$ and to not be in paid employment (OR= 1.75 ; $95 \%$ CI $1.09,2.81)$ or be in part-time employment $(\mathrm{OR}=1.76 ; 95 \%$ CI $1.03,3.00)$. No other significant differences between these two groups were observed.

The biological and sociodemographic characteristics of the cohort study are presented in Table 1 . The mean age of the participants was 31 years (range: 18-44 years). Slightly over half (53\%) of the participants were nulliparous and $30 \%$ of the participants were foreign nationals. The most common foreign nationalities represented in the study were Polish ( $8 \%$ ), followed by Chinese (2\%) and then British (1.8\%). Nearly half of the women (47\%) had obtained at least a degree qualification and over half (55\%) had private health insurance. About two-thirds (64\%) of the participants were married and the vast majority of participants ( $90 \%$ ) lived with the baby's father. The mean BMI of the sample was 23.9 (range: 15.0-49.9) $\mathrm{kg} / \mathrm{m}^{2}$, which is within the normal range.

The total weight gains of $10 \%$ of the sample were below the IOM (2009) recommendations. In addition, the 
Table 1 Biological and sociodemographic characteristics of the sample according to gestational weight gain (GWG): prospective cohort of pregnant women ( $n$ 799), recruited in March-May 2011 at the National Maternity Hospital, Dublin, Republic of Ireland

\begin{tabular}{|c|c|c|c|c|c|}
\hline Variable & Sample (\%) & Mean GWG (kg) & Inadequate GWG (\%) & Adequate GWG (\%) & Excessive GWG (\%) \\
\hline \multicolumn{6}{|l|}{ BMI class } \\
\hline Underweight $\left(<18.5 \mathrm{~kg} / \mathrm{m}^{2}\right)$ & 4.4 & $15 \cdot 8$ & $14 \cdot 3$ & 42.9 & $42 \cdot 9$ \\
\hline Normal weight $\left(18.5-24.9 \mathrm{~kg} / \mathrm{m}^{2}\right) \dagger$ & $64 \cdot 7$ & $16 \cdot 4$ & $10 \cdot 1$ & $32 \cdot 1$ & 57.8 \\
\hline Overweight $\left(25.0-29.9 \mathrm{~kg} / \mathrm{m}^{2}\right)$ & 20.5 & $16 \cdot 0$ & $6 \cdot 1$ & $12 \cdot 8$ & $81 \cdot 1^{\star}$ \\
\hline Obese $\left(\geq 30.0 \mathrm{~kg} / \mathrm{m}^{2}\right)$ & $10 \cdot 4$ & $12 \cdot 0^{*}$ & $15 \cdot 7$ & $21 \cdot 7$ & $62 \cdot 7$ \\
\hline \multicolumn{6}{|l|}{ Parity } \\
\hline $0+$ & $52 \cdot 8$ & $16 \cdot 9$ & $7 \cdot 3$ & $26 \cdot 3$ & $66 \cdot 4$ \\
\hline 1 & $29 \cdot 8$ & $15 \cdot 2^{*}$ & $9 \cdot 7$ & $30 \cdot 7$ & 59.7 \\
\hline $2+$ & $17 \cdot 4$ & $13 \cdot 9^{\star}$ & $18 \cdot 7^{*}$ & $25 \cdot 9$ & $55 \cdot 4^{\star}$ \\
\hline \multicolumn{6}{|l|}{ Age } \\
\hline $18-24$ years & $8 \cdot 4$ & $15 \cdot 6$ & $9 \cdot 0$ & $31 \cdot 3$ & $59 \cdot 7$ \\
\hline $25-29$ years $t$ & 24.5 & $16 \cdot 6$ & $8 \cdot 2$ & $25 \cdot 5$ & $66 \cdot 3$ \\
\hline 30-34 years & $43 \cdot 1$ & $15 \cdot 8$ & 9.9 & $27 \cdot 6$ & $62 \cdot 5$ \\
\hline $35+$ years & $24 \cdot 0$ & $15 \cdot 2^{*}$ & $12 \cdot 5$ & $28 \cdot 1$ & $59 \cdot 4$ \\
\hline \multicolumn{6}{|l|}{ Height } \\
\hline$<157 \mathrm{~cm}$ & $9 \cdot 1$ & $13 \cdot 8^{*}$ & 13.7 & $32 \cdot 9$ & $53 \cdot 4^{*}$ \\
\hline $157-170 \mathrm{~cm}$ & $71 \cdot 3$ & $15 \cdot 7^{*}$ & $9 \cdot 6$ & $29 \cdot 1$ & $61 \cdot 2^{*}$ \\
\hline$>170 \mathrm{~cm} \dagger$ & 19.5 & $17 \cdot 2$ & $9 \cdot 6$ & $19 \cdot 2$ & 71.2 \\
\hline \multicolumn{6}{|l|}{ Marital status } \\
\hline Married $†$ & $63 \cdot 8$ & $16 \cdot 0$ & $9 \cdot 6$ & 27.5 & $62 \cdot 9$ \\
\hline Single & $35 \cdot 8$ & $15 \cdot 6$ & $10 \cdot 8$ & $27 \cdot 3$ & 61.9 \\
\hline Unknown & 0.4 & & & & \\
\hline \multicolumn{6}{|l|}{ Lives with baby's father } \\
\hline Yest & $89 \cdot 6$ & $15 \cdot 9$ & $9 \cdot 6$ & $27 \cdot 2$ & $63 \cdot 1$ \\
\hline No & $9 \cdot 8$ & $15 \cdot 0$ & $14 \cdot 1$ & 29.5 & $56 \cdot 4$ \\
\hline Unknown & 0.6 & & & & \\
\hline \multicolumn{6}{|l|}{ Nationality } \\
\hline Irish national† & $70 \cdot 0$ & $15 \cdot 2$ & $12 \cdot 2$ & $28 \cdot 6$ & $59 \cdot 2$ \\
\hline Foreign national & $30 \cdot 0$ & $17 \cdot 3^{\star}$ & $5 \cdot 0^{*}$ & $25 \cdot 0$ & $70 \cdot 0^{*}$ \\
\hline \multicolumn{6}{|l|}{ Educational attainment } \\
\hline$<$ Second level & $5 \cdot 3$ & $13 \cdot 2^{*}$ & $16 \cdot 7$ & $26 \cdot 2$ & $57 \cdot 1$ \\
\hline Second level completed & 11.6 & $16 \cdot 0$ & 6.5 & $28 \cdot 0$ & $65 \cdot 6$ \\
\hline Vocational/training course & $35 \cdot 7$ & $15 \cdot 6$ & $10 \cdot 5$ & $27 \cdot 0$ & $62 \cdot 5$ \\
\hline Degree/postgraduate $†$ & $47 \cdot 2$ & $16 \cdot 3$ & 9.8 & 27.9 & $62 \cdot 3$ \\
\hline Unknown & 0.3 & & & & \\
\hline \multicolumn{6}{|l|}{ Employment } \\
\hline Not employed & $21 \cdot 3$ & $14 \cdot 3^{*}$ & $14 \cdot 7^{\star}$ & $27 \cdot 6$ & $57 \cdot 6$ \\
\hline Part-time employed & $15 \cdot 0$ & $15 \cdot 0^{*}$ & $10 \cdot 8$ & $30 \cdot 8$ & $58 \cdot 3$ \\
\hline Full-time employed $\dagger$ & $63 \cdot 6$ & $16 \cdot 6$ & 8.3 & $26 \cdot 8$ & $65 \cdot 0$ \\
\hline Unknown & 0.1 & & & & \\
\hline \multicolumn{6}{|l|}{ Pregnancy intention } \\
\hline Intended $\dagger$ & $74 \cdot 3$ & $16 \cdot 1$ & 8.9 & 27.4 & $63 \cdot 6$ \\
\hline Unintended & $25 \cdot 2$ & $15 \cdot 1^{*}$ & $13 \cdot 4$ & $27 \cdot 9$ & $58 \cdot 7$ \\
\hline Unknown & 0.5 & & & & \\
\hline \multicolumn{6}{|l|}{ Health insurance } \\
\hline Yes† & $55 \cdot 4$ & $16 \cdot 2$ & $10 \cdot 0$ & $27 \cdot 2$ & $62 \cdot 8$ \\
\hline No & $43 \cdot 8$ & $15 \cdot 3^{*}$ & 9.8 & $28 \cdot 6$ & $61 \cdot 6$ \\
\hline Unknown & 0.8 & & & & \\
\hline
\end{tabular}

${ }^{*} P<0.05$

†Reference category for univariable analysis of excessive and inadequate weight gain, respectively.

total weight gains of $27.5 \%$ of participants were within the IOM recommendations, while $62.5 \%$ exceeded the recommendations.

Tables 1 and 2 show the univariable associations between the categorised exposure variables and the outcome measures of GWG. The univariable analyses showed that all of the biological variables were significantly associated with at least one of the outcomes. For example, pre-pregnancy obesity, increased parity, short stature and older age (35+ years) were associated with lower weight gain. Additionally, overweight was a significant risk factor for excessive GWG, while multiparity was a risk factor for inadequate gain.
The univariable analyses showed that most of the sociodemographic variables were significantly related to GWG. For instance, unintended pregnancy, not completing second level education, absence of health insurance and not being in paid employment were associated with lower weight gain. Furthermore, foreign nationality was a significant risk factor for excessive weight gain, while not being employed was a risk factor for low weight gain.

The univariable analyses also showed that some of the health behaviour factors were significantly associated with GWG. Daily energy intake (as a continuous measure) was positively associated with weight gain. Increased food intake and smoking cessation were associated with higher 
Table 2 Health behaviour and psychological well-being characteristics of the sample according to gestational weight gain: prospective cohort of pregnant women ( $n$ 799), recruited in March-May 2011 at the National Maternity Hospital, Dublin, Republic of Ireland

\begin{tabular}{|c|c|c|c|c|c|}
\hline Variable & Sample (\%) & Mean GWG (kg) & Inadequate GWG (\%) & Adequate GWG (\%) & Excessive GWG (\%) \\
\hline \multicolumn{6}{|l|}{ Total daily energy intake } \\
\hline T1 (low) & 34.4 & 15.5 & $9 \cdot 8$ & $27 \cdot 6$ & $62 \cdot 5$ \\
\hline T2 (medium) $\dagger$ & $32 \cdot 7$ & $15 \cdot 8$ & $12 \cdot 3$ & $26 \cdot 8$ & $60 \cdot 9$ \\
\hline T3 (high) & $32 \cdot 3$ & $16 \cdot 3$ & 7.4 & $28 \cdot 3$ & $64 \cdot 3$ \\
\hline Unknown & 0.6 & & & & \\
\hline \multicolumn{6}{|c|}{ Changes in amount of food eaten since pregnant } \\
\hline A lot less & $7 \cdot 3$ & $12 \cdot 8^{*}$ & $17 \cdot 2$ & $29 \cdot 3$ & 53.4 \\
\hline A little less & $15 \cdot 1$ & $14 \cdot 0^{*}$ & 11.6 & $29 \cdot 8$ & $58 \cdot 7$ \\
\hline Same† & 24.0 & $15 \cdot 2$ & 13.5 & 29.2 & $57 \cdot 3$ \\
\hline A little more & $42 \cdot 8$ & $16 \cdot 9^{\star}$ & $7 \cdot 9^{\star}$ & $26 \cdot 6$ & 65.5 \\
\hline A lot more & $10 \cdot 4$ & $18 \cdot 0^{*}$ & $3.6^{*}$ & 22.9 & $73 \cdot 5^{\star}$ \\
\hline Unknown & 0.4 & & & & \\
\hline \multicolumn{6}{|c|}{ Takeaway consumption in pregnancy } \\
\hline Once weekly or lesst & 94.9 & $15 \cdot 8$ & $10 \cdot 2$ & $27 \cdot 7$ & $62 \cdot 1$ \\
\hline More than once weekly & $5 \cdot 1$ & $17 \cdot 4$ & $7 \cdot 3$ & 24.4 & $68 \cdot 3$ \\
\hline \multicolumn{6}{|c|}{ Fried food consumption per week in pregnancy } \\
\hline $0-3 \dagger$ & 92.5 & $15 \cdot 8$ & $10 \cdot 3$ & $27 \cdot 2$ & 62.5 \\
\hline $4+$ & $7 \cdot 0$ & $16 \cdot 0$ & $7 \cdot 1$ & 33.9 & $58 \cdot 9$ \\
\hline Unknown & 0.5 & & & & \\
\hline \multicolumn{6}{|c|}{ Number of snacks per day in pregnancy } \\
\hline $0-2 \dagger$ & 79.5 & $15 \cdot 7$ & $9 \cdot 4$ & $28 \cdot 8$ & $61 \cdot 7$ \\
\hline $3+$ & $20 \cdot 5$ & $16 \cdot 6$ & $12 \cdot 2$ & $22 \cdot 6$ & $65 \cdot 2$ \\
\hline \multicolumn{6}{|l|}{ Alcohol use in pregnancy } \\
\hline Yest & $18 \cdot 9$ & $15 \cdot 3$ & 11.9 & $31 \cdot 1$ & $57 \cdot 0$ \\
\hline No & $81 \cdot 1$ & $16 \cdot 0$ & 9.6 & $26 \cdot 7$ & 63.7 \\
\hline \multicolumn{6}{|c|}{ Change in physical activity since pregnant } \\
\hline A lot less active & $18 \cdot 1$ & $16 \cdot 4$ & $10 \cdot 3$ & $28 \cdot 3$ & 61.4 \\
\hline A little less active & 39.7 & $16 \cdot 1$ & $8 \cdot 8$ & 28.4 & $62 \cdot 8$ \\
\hline Same† & 37.5 & 15.4 & 11.3 & $26 \cdot 0$ & $62 \cdot 7$ \\
\hline A little more active & 3.6 & $16 \cdot 4$ & 3.4 & 31.0 & 65.5 \\
\hline A lot more active & 0.4 & $12 \cdot 6$ & 0.0 & 33.3 & $66 \cdot 7$ \\
\hline Unknown & 0.6 & & & & \\
\hline \multicolumn{6}{|l|}{ Hours of sleep per night } \\
\hline$<7$ & 13.9 & $15 \cdot 3$ & $15 \cdot 3^{*}$ & $27 \cdot 0$ & 57.7 \\
\hline$\geq 7 \dagger$ & $85 \cdot 5$ & $16 \cdot 0$ & 8.9 & 27.5 & 63.5 \\
\hline Ūnknown & 0.1 & & & & \\
\hline \multicolumn{6}{|c|}{ Smoking status in pregnancy } \\
\hline Current smoker & $7 \cdot 0$ & $13 \cdot 7^{*}$ & $16 \cdot 1$ & $30 \cdot 4$ & $53 \cdot 6$ \\
\hline Former & $19 \cdot 4$ & $17 \cdot 0^{*}$ & $9 \cdot 7$ & $20 \cdot 6$ & $69 \cdot 7$ \\
\hline Non-smoker† & 73.5 & $15 \cdot 8$ & 9.5 & $29 \cdot 1$ & $61 \cdot 3$ \\
\hline Unknown & 0.1 & & & & \\
\hline \multicolumn{6}{|c|}{ Leisure-time physical activity } \\
\hline T1 (low) & $32 \cdot 9$ & $15 \cdot 2$ & 11.5 & $26 \cdot 8$ & $61 \cdot 7$ \\
\hline T2 (moderate)† & 35.5 & $16 \cdot 1$ & 8.9 & 28.9 & $62 \cdot 1$ \\
\hline T3 (high) & $31 \cdot 3$ & $16 \cdot 2$ & 9.5 & $26 \cdot 6$ & 63.9 \\
\hline Unknown & 0.3 & & & & \\
\hline \multicolumn{6}{|l|}{ Antenatal depression } \\
\hline EPDS $>12$ & 14.5 & $15 \cdot 2$ & $10 \cdot 3$ & $35 \cdot 3$ & $54 \cdot 3$ \\
\hline $\mathrm{EPDS} \leq 12 \dagger$ & 85.5 & $16 \cdot 0$ & $10 \cdot 0$ & $26 \cdot 2$ & $63 \cdot 8$ \\
\hline \multicolumn{6}{|l|}{ Stress } \\
\hline T1 (low) & $34 \cdot 7$ & $16 \cdot 2$ & 11.6 & $23 \cdot 1$ & $65 \cdot 3$ \\
\hline T2 (moderate) $\dagger$ & $30 \cdot 8$ & $15 \cdot 8$ & $7 \cdot 3$ & 29.7 & $63 \cdot 0$ \\
\hline T3 (high) & 34.5 & $15 \cdot 6$ & $10 \cdot 9$ & $30 \cdot 1$ & $59 \cdot 1$ \\
\hline \multicolumn{6}{|l|}{ Optimism } \\
\hline T1 (low) & $32 \cdot 9$ & $15 \cdot 2$ & $11 \cdot 0$ & $29 \cdot 3$ & $59 \cdot 7$ \\
\hline T2 (moderate)† & 35.5 & $16 \cdot 1$ & $10 \cdot 6$ & $26 \cdot 1$ & 63.4 \\
\hline T3 (high) & $31 \cdot 3$ & $16 \cdot 2$ & 8.0 & $27 \cdot 6$ & 64.4 \\
\hline Unknown & 0.3 & & & & \\
\hline \multicolumn{6}{|l|}{ Prenatal distress } \\
\hline T1 (low) & $31 \cdot 0$ & $15 \cdot 7$ & $11 \cdot 7$ & $25 \cdot 0$ & $63 \cdot 3$ \\
\hline T2 (moderate)† & 34.4 & $15 \cdot 5$ & 9.8 & 34.2 & $56 \cdot 0$ \\
\hline T3 (high) & 34.0 & $16 \cdot 4$ & $8 \cdot 8$ & $22 \cdot 8$ & 68.4 \\
\hline
\end{tabular}

EPDS, Edinburgh Postpartum Depression Scale.

${ }^{*} P<0.05$.

†Reference category for univariable analysis of excessive and inadequate weight gain, respectively. 
weight gain, while decreased food intake and smoking during pregnancy were associated with lower weight gain. Eating a lot more food was related to excessive GWG, while sleeping for less than $7 \mathrm{~h}$ per night was associated with inadequate GWG.

None of the psychological variables were significantly associated with total weight gain or weight gain adequacy in the univariable analyses. Some of the psychological variables were however retained for further regression analyses, as the associated $P$ value for one or more categories of these variables was below 0 15 .

The final multivariable models of total GWG are shown in Table 3. The final model accounted for $23 \%$ of the variance in total weight gain. None of the psychological variables were independently related to weight gain. The final model showed that obese mothers gained about $3 \mathrm{~kg}$ less than normal-weight mothers. Additionally, increased parity, short stature and absence of health insurance were independently associated with lower weight gain, while foreign nationality was associated with higher weight gain. Two of the health behaviour factors, change in amount of food intake and takeaway consumption, were independently associated with total weight gain. Eating 'a lot less' food was associated with $1.5 \mathrm{~kg}$ less weight gain, while eating 'a little more' food was associated with $1.3 \mathrm{~kg}$ more weight gain. Furthermore, eating 'a lot more' food was associated with $2.7 \mathrm{~kg}$ greater weight gain. Finally, consuming more than one takeaway meal per week was associated with over $2 \mathrm{~kg}$ higher weight gain.

The model-building process showed that total energy intake (as a continuous measure) was positively associated with total weight gain following adjustments for nondietary risk factors. It was not considered appropriate to include the other dietary factors (takeaway consumption and change in food intake) in this model because the change in food intake measure may be considered a proxy for energy intake ${ }^{(6)}$ and because takeaway consumption may mediate the relationship between energy intake and GWG. Change in food intake and takeaway consumption were included in the final model of total weight gain (shown in Table 3), as these variables had a stronger impact on GWG than energy intake, as evidenced by the higher proportion of variance accounted for by this model (23\%) than the model including total energy intake (19\%).

Table 4 shows the final multivariable models of excessive weight gain and inadequate weight gain. Compared with normal-weight women, underweight women had significantly lower odds of exceeding the IOM guidelines, while both overweight and obese women had significantly higher odds of exceeding the guidelines. Conversely, overweight women had about $50 \%$ lower odds of inadequate weight gain. Multiparity was both protective against excessive gain and a significant risk factor for inadequate weight gain. Compared with tall women, both short and medium height women had significantly lower odds of exceeding the IOM guidelines. The adjusted models also
Table 3 Final multivariable regression model of total gestational weight gain: prospective cohort of pregnant women ( $n$ 799), recruited in March-May 2011 at the National Maternity Hospital, Dublin, Republic of Ireland

\begin{tabular}{|c|c|c|c|}
\hline Variable & $B$ & SE & $P$ value* \\
\hline \multicolumn{4}{|l|}{ BMI class } \\
\hline Underweight $\left(<18.5 \mathrm{~kg} / \mathrm{m}^{2}\right)$ & -0.95 & 0.89 & 0.285 \\
\hline Normal weight $\left(18.5-24.9 \mathrm{~kg} / \mathrm{m}^{2}\right)$ & Ref. & - & \\
\hline Overweight $\left(25.0-29.9 \mathrm{~kg} / \mathrm{m}^{2}\right)$ & 0.40 & 0.47 & 0.393 \\
\hline Obese $\left(\geq 30.0 \mathrm{~kg} / \mathrm{m}^{2}\right)$ & -3.05 & 0.65 & $<0.001$ \\
\hline \multicolumn{4}{|l|}{ Parity } \\
\hline 0 & Ref. & - & \\
\hline 1 & -0.97 & 0.42 & 0.021 \\
\hline $2+$ & -1.60 & 0.51 & 0.002 \\
\hline \multicolumn{4}{|l|}{ Height } \\
\hline$<157 \mathrm{~cm}$ & $-2 \cdot 31$ & 0.74 & 0.002 \\
\hline $157-170 \mathrm{~cm}$ & -0.80 & 0.47 & 0.089 \\
\hline$>170 \mathrm{~cm}$ & Ref. & - & \\
\hline \multicolumn{4}{|l|}{ Health insurance } \\
\hline Yes & Ref. & - & \\
\hline No & -1.04 & 0.38 & 0.007 \\
\hline \multicolumn{4}{|l|}{ Nationality } \\
\hline Irish national & Ref. & - & \\
\hline Foreign national & 1.71 & 0.42 & $<0.001$ \\
\hline \multicolumn{4}{|c|}{ Change in amount of food eaten since pregnant } \\
\hline A lot less & -1.53 & 0.77 & 0.046 \\
\hline A little less & -0.53 & 0.60 & 0.372 \\
\hline About the same & Ref. & - & \\
\hline A little more & 1.31 & 0.46 & 0.005 \\
\hline A lot more & 2.66 & 0.67 & $<0.001$ \\
\hline \multicolumn{4}{|l|}{ Takeaway consumption in pregnancy } \\
\hline Once weekly or less & Ref & - & \\
\hline More than once weekly & $2 \cdot 20$ & 0.82 & 0.007 \\
\hline
\end{tabular}

Ref., reference category.

Model controlled for length of gestation (weeks) and the length of time (weeks) between the last measured maternal weight and delivery. Model $R^{2}=0.23$.

${ }^{*} P<0.05$ is indicated in bold.

showed that foreign-born mothers had about double the odds of excessive weight gain and about $60 \%$ lower odds of inadequate weight gain. In addition, eating 'a little more' food increased the odds of excessive weight gain by about $60 \%$, while eating 'a lot more' food more than doubled the odds of excessive weight gain. Finally, probable antenatal depression was associated with decreased odds of exceeding the IOM guidelines.

\section{Discussion}

Guided by the biopsychosocial model, the present analysis examined whether potentially modifiable health behaviour and psychological well-being measures were associated with GWG, when relatively unmodifiable biological and sociodemographic measures were taken into account. The analysis identified two potentially modifiable variables (pregnancy-associated change in food intake and takeaway consumption) that were associated with GWG in a biopsychosocial model.

The association of biological characteristics with GWG was largely consistent with previous research. The finding that obese women gained about $3 \mathrm{~kg}$ less on average than 
Table 4 Final multivariable logistic regression models predicting excessive weight gain and inadequate weight gain, respectively: prospective cohort of pregnant women ( $n$ 799), recruited in March-May 2011 at the National Maternity Hospital, Dublin, Republic of Ireland

\begin{tabular}{|c|c|c|c|c|c|c|}
\hline \multirow[b]{2}{*}{ Variable } & \multicolumn{3}{|c|}{ Model of excessive weight gain $v$. not excessive } & \multicolumn{3}{|c|}{ Model of inadequate weight gain $v$. not inadequate } \\
\hline & OR & $95 \% \mathrm{Cl}$ & $P$ value* & OR & $95 \% \mathrm{Cl}$ & $P$ value $^{*}$ \\
\hline \multicolumn{7}{|l|}{ BMl class } \\
\hline Underweight $\left(<18.5 \mathrm{~kg} / \mathrm{m}^{2}\right)$ & 0.41 & $0.20,0.86$ & 0.018 & 1.97 & $0.71,5.53$ & 0.196 \\
\hline Normal weight $\left(18.5-24.9 \mathrm{~kg} / \mathrm{m}^{2}\right)$ & Ref. & - & & Ref. & - & \\
\hline Overweight $\left(25 \cdot 0-29 \cdot 9 \mathrm{~kg} / \mathrm{m}^{2}\right)$ & 4.43 & $2 \cdot 80,6 \cdot 99$ & $<0.001$ & 0.48 & $0.23,0.98$ & 0.043 \\
\hline Obese $\left(\geq 30.0 \mathrm{~kg} / \mathrm{m}^{2}\right)$ & $2 \cdot 12$ & $1 \cdot 24,3 \cdot 61$ & 0.006 & 0.99 & $0.48,2.03$ & 0.978 \\
\hline \multicolumn{7}{|l|}{ Parity } \\
\hline 0 & Ref. & - & & Ref. & - & \\
\hline 1 & 0.76 & $0.53,1.08$ & 0.123 & 1.26 & $0.70,2.25$ & 0.444 \\
\hline $2+$ & 0.64 & $0.42,0.98$ & 0.041 & 2.59 & $1.43,4.69$ & 0.002 \\
\hline \multicolumn{7}{|l|}{ Height } \\
\hline$<157 \mathrm{~cm}$ & 0.38 & $0.20,0.72$ & 0.003 & & & \\
\hline $157-170 \mathrm{~cm}$ & 0.62 & $0.41,0.93$ & 0.021 & & & \\
\hline$>170 \mathrm{~cm}$ & Ref. & - & & & & \\
\hline \multicolumn{7}{|l|}{ Nationality } \\
\hline Irish national & Ref. & - & & Ref. & - & \\
\hline Foreign national & 1.93 & $1 \cdot 36,2 \cdot 75$ & $<0.001$ & 0.41 & $0.21,0.79$ & 0.008 \\
\hline \multicolumn{7}{|c|}{ Change in amount of food eaten since pregnant } \\
\hline A lot less & 0.93 & $0.49,1 \cdot 76$ & 0.816 & $1 \cdot 15$ & $0.50,2.64$ & 0.748 \\
\hline A little less & 0.90 & $0.58,1.58$ & 0.863 & 0.77 & $0.38,1.60$ & 0.489 \\
\hline About the same & Ref. & - & & Ref. & - & \\
\hline A little more & 1.64 & $1.11,2.42$ & 0.012 & 0.55 & $0.31,0.99$ & 0.045 \\
\hline A lot more & $2 \cdot 57$ & $1.42,4.66$ & 0.002 & 0.21 & $0.06,0.74$ & 0.014 \\
\hline \multicolumn{7}{|l|}{ Antenatal depression } \\
\hline EPDS > 12 & Ref. & - & & & & \\
\hline EPDS $\leq 12$ & 0.62 & $0.40,0.96$ & 0.032 & & & \\
\hline
\end{tabular}

EPDS, Edinburgh Postpartum Depression Scale; Ref., reference category.

${ }^{*} P<0.05$ is indicated in bold.

normal-weight women, but still had higher odds of exceeding the weight gain guidelines, is compatible with previous research ${ }^{(6)}$. This finding may seem contradictory at first, but it is explained by the fact that the IOMrecommended GWG range for obese women $(5 \cdot 0-9 \cdot 0 \mathrm{~kg})$ is substantially lower than the recommended range for normal-weight women $(11.5-16.0 \mathrm{~kg})^{(1)}$. Furthermore, there was no significant difference between overweight and normal-weight women in absolute weight gain. Indeed, pre-pregnancy overweight was the strongest risk factor for exceeding the IOM guidelines in the present research. The overweight women may have been unaware that they should be gaining less than normal-weight women, as there are currently no prenatal weight gain recommendations in Ireland. Nevertheless, in spite of the existence of lower weight gain recommendations for overweight women (than normal-weight women) in the USA since 1990, numerous US studies have found that overweight women have the highest risk of exceeding the recommendations of all BMI classes ${ }^{(6,8,16,17)}$.

The finding that parous women had lower GWG has been previously documented ${ }^{(16,47,48)}$. In addition, the finding that multiparity was both protective against excessive weight gain and a risk factor for inadequate weight gain is consistent with a number of prior studies ${ }^{(5,16,17)}$. Also compatible with previous research is the observation that short women gained significantly less weight and had lower odds of exceeding the IOM guidelines $^{(49-51)}$. However, the present research did not find evidence that short stature was a risk factor for inadequate weight gain. The evidence base is mixed regarding the relationship between short stature and insufficient weight gain. Some studies have found that short stature is a risk factor for insufficient weight gain $^{(49,51,52)}$, while others have not ${ }^{(17,53)}$. Finally, the present study observed that women aged 35 years or above had significantly lower maternal weight gain in the unadjusted analysis, but that following adjustment for other risk factors, this relationship was no longer significant. These findings contradict some studies, which have shown an independent association between older maternal age and lower $\mathrm{GWG}^{(47,49)}$.

Some of the sociodemographic characteristics were independently associated with the weight gain outcomes. The finding that women without private health insurance had significantly lower total weight gain in the multivariable model is consistent with previous research showing that indicators of lower socio-economic status (such as lower income and education) are associated with lower weight gain $^{(9,47)}$. The multivariable models also indicated that foreign nationals have an increased risk of excessive weight gain and conversely Irish nationals have an increased risk of inadequate weight gain. As excessive weight gain is very common among both foreign-born and 
Irish-born mothers, both groups may need more information about the health risks of excessive weight gain, while messages about the risks of inadequate weight gain may be especially pertinent for Irish-born mothers ${ }^{(54,55)}$.

Some of the health behaviour measures predicted GWG. To our knowledge, the present study is the first one to find an independent association between consumption of takeaway meals and GWG. Additionally, the significant relationships observed between the self-reported change in food intake measure and GWG are consistent with research by Olson and Strawderman ${ }^{(6)}$ using a similar measure, which showed that eating 'a lot more' food during pregnancy was independently associated with both higher total weight gain and excessive weight gain. The present research, however, is the first to show that women who reported eating 'a little more' food during pregnancy had both an increased risk of excessive weight gain and a decreased risk of inadequate weight gain. These findings suggest that health messages about food intake during pregnancy need to carefully balance the competing risks of excessive and inadequate weight gain.

Total energy intake was significantly associated with increased absolute weight gain following adjustment for non-dietary confounding factors. However, takeaway consumption and change in food intake were the only dietary variables included in the final model as they accounted for more variance in GWG than energy intake. This suggests that single-item measures of dietary intake, particularly change in food intake, may be more effective at predicting GWG than a longer FFQ.

Many of the behavioural measures were not independently associated with GWG. The present findings are consistent with prior studies which have shown that alcohol use $^{(56)}$ and sleep duration ${ }^{(14)}$ in pregnancy are not associated with GWG. Smoking during pregnancy was associated with lower total weight gain in the univariable analysis; however, this effect was no longer significant following adjustment for other risk factors. Some studies investigating the relationship between smoking during pregnancy and maternal weight gain have shown significant results ${ }^{(6,15)}$, while other have not ${ }^{(16,17,52)}$. Studies examining the relationship between pregnancy-related smoking cessation and GWG have yielded more consistent results ${ }^{(15,52,57)}$. None the less, the present study did not observe a significant association between pregnancyrelated smoking cessation and GWG, following adjustment for confounders.

Consistent with previous studies using a biopsychsocial approach, most of the psychological well-being measures were not associated with GWG, following adjustments for behavioural, sociodemographic and biological variables $^{(6,11)}$. The present research showed however that antenatal depression was protective against excessive weight gain, following adjustment for other risk factors. Nevertheless, depressed mood was not associated with an increased risk of inadequate weight gain. In contrast, a number of previous studies have observed a significant relationship between depressive symptoms and low weight gain ${ }^{(50,58-60)}$.

Strengths of the research include a large sample size and a prospective design. A limitation of the study is that information on nausea and vomiting was not collected in the self-administered questionnaire, as these data may have explained the relationship between eating 'a lot less food' and lower GWG. A second limitation is that the women excluded from the analysis due to missing weight information were of lower socio-economic status than those included in the analysis with regard to employment and health insurance status. No differences in terms of biological, well-being or behavioural factors were observed, however, which suggests that the exclusions did not substantially bias the study findings. Another limitation of the research is that physical activity was assessed using self-report measurements rather than objective measurements (such as accelerometry) ${ }^{(61)}$. The use of self-report methods may explain why neither leisure-time physical activity scores nor changes in physical activity levels since becoming pregnant were associated with GWG. Several reviews have concluded that physical activity levels during pregnancy did not impact on GWG, or at least not in most studies ${ }^{(13,62-64)}$. These reviews were based mainly on observational studies and physical activity levels were usually assessed using a questionnaire. However, a recent meta-analysis of intervention trials found lower mean GWG among the intervention group than the controls ${ }^{(65)}$. On the whole, it seems that future research may need to use objective measures of physical activity to accurately examine the relationship between physical activity levels and GWG.

As the sample was large and consecutively recruited and because a large variety of factors were examined simultaneously, including nationality, these results may be widely generalisable to non-diabetic mothers with singleton pregnancies attending antenatal clinics in westernised countries. In addition, most studies of this kind have been conducted with US samples, and thus the similarity of the present results with previous findings enhances the generalisability of our analysis.

\section{Conclusions}

A variety of biological, sociodemographic, behavioural and affective measures were associated with GWG in a biopsychosocial model. Indeed, a clinically relevant proportion of the variance in total weight gain (23\%) was predicted by the final biopsychosocial model of total GWG. We identified two potentially modifiable behaviours that influenced GWG: takeaway consumption and pregnancy-associated change in food intake. The findings suggest that women are aware in early pregnancy whether they have drastically increased their food intake and this 
suggests that early pregnancy might be an opportune time to identify such women and to advise them about appropriate energy requirements in pregnancy. In addition, pregnant women could be advised that energy-dense meals, such as takeaways, should not be consumed on a frequent basis.

We also identified a number of relatively unmodifiable factors that influenced GWG. These findings highlight subgroups of women, including nulliparous, foreign national and high BMI women, who could be targeted for inclusion in weight-management interventions during or after pregnancy. Nevertheless, a very high prevalence of excessive weight gain was identified, and therefore all women entering antenatal care in Ireland may need advice about continuity in healthy eating habits across pregnancy and the postpartum period.

\section{Acknowledgements}

Financial support: This research was supported by a grant (HRC 2007/13) from the Health Research Board, Dublin, Ireland. The Health Research Board had no role in the design, analysis or writing of this article. Conflict of interest: None. Authorship: E.H. designed the study, coordinated data collection, conducted the analysis and drafted the paper. C.C.K. and P.G.W. advised on data analysis, interpretation of findings and revision of the paper. F.M.M. contributed to study design, data analysis and interpretation, and drafting of the paper. Each author read and approved the content of the final manuscript. Ethics of buman subject participation: This study was approved by the Ethics Committee of the National Maternity Hospital, Dublin, Ireland.

\section{References}

1. Institute of Medicine (2009) Weight Gain During Pregnancy: Reexamining the Guidelines. Washington, DC: The National Academies Press.

2. Viswanathan M, Siega-Riz AM, Moos MK et al. (2008) Outcomes of maternal weight gain. Evid Rep Technol Assess (Full Rep) 168, 1-223.

3. Siega-Riz AM, Viswanathan M, Moos MK et al. (2009) A systematic review of outcomes of maternal weight gain according to the Institute of Medicine recommendations: birthweight, fetal growth, and postpartum weight retention. Am J Obstet Gynecol 201, 339.e1-e14.

4. Nehring I, Lehmann S \& von Kries R (2013) Gestational weight gain in accordance to the IOM/NRC criteria and the risk for childhood overweight: a meta-analysis. Pediatr Obes 8, 218-224.

5. Wells CS, Schwalberg R, Noonan G et al. (2006) Factors influencing inadequate and excessive weight gain in pregnancy: Colorado, 2000-2002. Matern Child Health J 10, 55-62.

6. Olson CM \& Strawderman MS (2003) Modifiable behavioral factors in a biopsychosocial model predict inadequate and excessive gestational weight gain. J Am Diet Assoc 103, $48-54$.
7. Strychar IM, Chabot C, Champagne F et al. (2000) Psychosocial and lifestyle factors associated with insufficient and excessive maternal weight gain during pregnancy. J Am Diet Assoc 100, 353-356.

8. Weisman CS, Hillemeier MM, Downs DS et al. (2010) Preconception predictors of weight gain during pregnancy: prospective findings from the Central Pennsylvania Women's Health Study. Womens Health Issues 20, 126-132.

9. Hickey CA (2000) Sociocultural and behavioral influences on weight gain during pregnancy. Am J Clin Nutr 71, 5 Suppl., 1364S-1370S.

10. Olson CM, Strawderman MS, Hinton PS et al. (2003) Gestational weight gain and postpartum behaviors associated with weight change from early pregnancy to $1 \mathrm{y}$ postpartum. Int J Obes Relat Metab Disord 27, 117-127.

11. Webb JB, Siega-Riz AM \& Dole N (2009) Psychosocial determinants of adequacy of gestational weight gain. Obesity (Silver Spring) 17, 300-309.

12. Streuling I, Beyerlein A, Rosenfeld E et al. (2011) Weight gain and dietary intake during pregnancy in industrialized countries - a systematic review of observational studies. J Perinat Med 39, 123-129.

13. Schlüssel MM, Souza EB, Reichenheim ME et al. (2008) Physical activity during pregnancy and maternal-child health outcomes: a systematic literature review. Cad Saude Publica 24, Suppl. 4, s531-s544.

14. Herring SJ, Nelson DB, Davey A et al. (2012) Determinants of excessive gestational weight gain in urban, lowincome women. Womens Health Issues 22, e439-e446.

15. Olafsdottir AS, Skuladottir GV, Thorsdottir I et al. (2006) Combined effects of maternal smoking status and dietary intake related to weight gain and birth size parameters. $\mathrm{BrJ}$ Obstet Gynaecol 113, 1296-1302.

16. Chasan-Taber L, Schmidt MD, Pekow P et al. (2008) Predictors of excessive and inadequate gestational weight gain in Hispanic women. Obesity (Silver Spring) 16, 1657-1666.

17. Walker LO, Hoke MM \& Brown A (2009) Risk factors for excessive or inadequate gestational weight gain among Hispanic women in a US-Mexico border state. $J$ Obstet Gynecol Neonatal Nurs 38, 418-429.

18. Mehta UJ, Siega-Riz AM \& Herring AH (2011) Effect of body image on pregnancy weight gain. Matern Child Health J 15, 324-332.

19. Rasmussen KM, Abrams B, Bodnar LM et al. (2010) Recommendations for weight gain during pregnancy in the context of the obesity epidemic. Obstet Gynecol 116, 1191-1195.

20. World Health Organisation (2002) Diet, Nutrition and the Prevention of Chronic Diseases. Joint WHO/FAO Expert Consultation WHO Technical Report Series no. 916. Geneva: WHO

21. Oken E, Taveras EM, Kleinman KP et al. (2007) Gestational weight gain and child adiposity at age 3 years. Am J Obstet Gynecol 196, 322.e1-e8.

22. Stevens-Simon C, McAnarney ER \& Coulter MP (1986) How accurately do pregnant adolescents estimate their weight prior to pregnancy? J Adolesc Health Care 7, 250-254.

23. Stevens-Simon C, Roghmann KJ \& McAnarney ER (1992) Relationship of self-reported prepregnant weight and weight gain during pregnancy to maternal body habitus and age. J Am Diet Assoc 92, 85-87.

24. Laraia BA, Siega-Riz AM \& Gundersen C (2010) Household food insecurity is associated with self-reported pregravid weight status, gestational weight gain, and pregnancy complications. J Am Diet Assoc 110, 692-701.

25. Siega-Riz AM, Adair LS \& Hobel CJ (1994) Institute of Medicine maternal weight gain recommendations and pregnancy outcome in a predominantly Hispanic population. Obstet Gynecol 84, 565-573. 
26. Centers for Disease Control and Prevention (2013) Pregnancy Risk Assessment Monitoring System, What is PRAMS? http://www.cdc.gov/prams/ (accessed June 2013).

27. Cox JL, Chapman G, Murray D et al. (1996) Validation of the Edinburgh Postnatal Depression scale (EPDS) in nonpostnatal women. J Affect Disord 39, 185-189.

28. Cox JL, Holden JM \& Sagovsky R (1987) Detection of postnatal depression - development of the 10-item Edinburgh Postnatal Depression scale. Br J Psychiatry 150, 782-786.

29. Evans J, Heron J, Francomb H et al. (2001) Cohort study of depressed mood during pregnancy and after childbirth. BMJ 323, 257-260.

30. Cohen S (1988) Perceived stress in a probability sample of the United States. In The Social Psychology of Health, pp. 31-67 [S Spacapan and S Oskamp, editors]. Thousand Oaks, CA: Sage Publications, Inc.

31. Roberti J, Harrington L \& Storch E (2006) Further psychometric support for the 10-item version of the Perceived Stress scale. J Coll Counsel 9, 135-147.

32. Yali AM \& Lobel M (1999) Coping and distress in pregnancy: an investigation of medically high risk women. J Psychosom Obstet Gynaecol 20, 39-52.

33. Lobel M, Cannella DL, Graham JE et al. (2008) Pregnancyspecific stress, prenatal health behaviors, and birth outcomes. Health Psychol 27, 604-615.

34. Scheier MF, Carver CS \& Bridges MW (1994) Distinguishing optimism from neuroticism (and trait anxiety, self-mastery and self-esteem): a reevaluation of the Life Orientation test. J Pers Soc Psychol 67, 1063-1078.

35. Cox JL \& Holden JM (2003) Perinatal Mental Health: A Guide to the Edinburgh Postnatal Depression Scale (EPDS). London: Gaskell.

36. National Nutritional Surveillance Centre (2003) Dietary Habits of the Irish Population: Results from SLÁN, Annual Report 2003. Dublin: National Nutritional Surveillance Centre, Department of Public Health Medicine and Epidemiology, University College Dublin and Health Promotion Unit, Department of Health and Children.

37. Bingham SA, Gill C, Welch A et al. (1997) Validation of dietary assessment methods in the UK arm of EPIC using weighed records, and 24-hour urinary nitrogen and potassium and serum vitamin $\mathrm{C}$ and carotenoids as biomarkers. Int J Epidemiol 26, Suppl. 1, S137-S151.

38. Harrington J (1997) Validation of a food frequency questionnaire as a tool for assessing nutrient intakes. MA Thesis, National University of Ireland, Galway.

39. Godin G \& Shephard RJ (1985) A simple method to assess exercise behavior in the community. Can J Appl Sport Sci 10, 141-146.

40. Sallis JF, Buono MJ, Roby JJ et al. (1993) Seven-day recall and other physical activity self-reports in children and adolescents. Med Sci Sports Exerc 25, 99-108.

41. Jacobs DR, Ainsworth BE, Hartman TJ et al. (1993) A simultaneous evaluation of 10 commonly used physical activity questionnaires. Med Sci Sports Exerc 25, 81-91.

42. Miller DJ, Freedson PS \& Kline GM (1994) Comparison of activity levels using the Caltrac accelerometer and five questionnaires. Med Sci Sports Exerc 26, 376-382.

43. Hinton PS \& Olson CM (2001) Postpartum exercise and food intake: the importance of behavior-specific self-efficacy. J Am Diet Assoc 101, 1430-1437.

44. Gangwisch JE, Malaspina D, Boden-Albala B et al. (2005) Inadequate sleep as a risk factor for obesity: analyses of the NHANES I. Sleep 28, 1289-1296.

45. Kirkwood BR \& Stern JL (2005) Essential Medical Statistics, 2nd ed. Oxford: Blackwell Science Ltd.

46. Matthews JNS (2005) Model, choice of. In Encyclopedia of Biostatistics. Chichester: John Wiley \& Sons, Ltd; available at http://onlinelibrary.wiley.com/doi/10.1002/0470011815.b2a 09030/abstract

47. Chu SY, Callaghan WM, Bish CL et al. (2009) Gestational weight gain by body mass index among US women delivering live births, 2004-2005: fueling future obesity. Am J Obstet Gynecol 200, 271.e1-e7.

48. Harris HE, Ellison GTH \& Holliday M (1997) Is there an independent association between parity and maternal weight gain? Ann Hum Biol 24, 507-519.

49. Siega-Riz AM \& Adair LS (1993) Biological determinants of pregnancy weight gain in a Filipino population. Am J Clin Nutr 57, 365-372.

50. Siega-Riz AM \& Hobel CJ (1997) Predictors of poor maternal weight gain from baseline anthropometric, psychosocial, and demographic information in a Hispanic population. J Am Diet Assoc 97, 1264-1268.

51. Caulfield LE, Witter FR \& Stoltzfus RJ (1996) Determinants of gestational weight gain outside the recommended ranges among black and white women. Obstet Gynecol 87, $760-766$.

52. Rodrigues PL, Costa de Oliveira L, Santos Brito A et al. (2010) Determinant factors of insufficient and excessive gestational weight gain and maternal-child adverse outcomes. Nutrition 26, 617-623.

53. Institute of Medicine (1990) Nutrition During Pregnancy. Washington, DC: National Academies Press.

54. Heery E, McConnon A, Kelleher CC et al. (2013) Perspectives on weight gain and lifestyle practices during pregnancy among women with a history of macrosomia: a qualitative study in the Republic of Ireland. BMC Pregnancy Childbirth 13, 202.

55. Groth SW \& Kearney MH (2009) Diverse women's beliefs about weight gain in pregnancy. J Midwifery Womens Health 54, 452-457.

56. Little RE, Asker RL, Sampson PD et al. (1986) Fetal growth and moderate drinking in early pregnancy. Am J Epidemiol 123, 270-278.

57. Mongoven M, Dolan-Mullen P, Groff JY et al. (1996) Weight gain associated with prenatal smoking cessation in white, non-Hispanic women. Am J Obstet Gynecol 174, $72-77$.

58. Zuckerman B, Amaro H, Bauchner H et al. (1989) Depressive symptoms during pregnancy: relationship to poor health behaviors. Am J Obstet Gynecol 160, 1107-1111

59. Bodnar LM, Wisner KL, Moses-Kolko E et al. (2009) Prepregnancy body mass index, gestational weight gain, and the likelihood of major depressive disorder during pregnancy. J Clin Psychiatry 70, 1290-1296.

60. Hickey CA, Cliver SP, Goldenberg RL et al. (1995) Relationship of psychosocial status to low prenatal weight gain among nonobese black and white women delivering at term. Obstet Gynecol 86, 177-183.

61. Bell R, Tennant PWG, McParlin C et al. (2013) Measuring physical activity in pregnancy: a comparison of accelerometry and self-completion questionnaires in overweight and obese women. Eur J Obstet Gynecol Reprod Biol 170, 90-95.

62. Lokey EA, Tran ZV, Wells CL et al. (1991) Effects of physical exercise on pregnancy outcomes: a meta-analytic review. Med Sci Sports Exerc 23, 1234-1239.

63. Stevenson L (1997) Exercise in pregnancy. Part 1: Update on pathophysiology. Can Fam Physician 43, 97-104.

64. Morris SN \& Johnson NR (2005) Exercise during pregnancy - a critical appraisal of the literature. J Reprod Med 50, 181-188.

65. Streuling I, Beyerlein A, Rosenfeld E et al. (2011) Physical activity and gestational weight gain: a meta-analysis of intervention trials. BrJ Obstet Gynaecol 118, 278-284. 